\title{
Investigation and Reflection on Medical Treatment of International Students in Nanchong
}

\author{
Hongxia Zhao \\ Department of Foreign Languages and Cultures, North Sichuan Medical College, Nanchong, China \\ Email: 2926966208@qq.com
}

How to cite this paper: Zhao, H.X. (2021) Investigation and Reflection on Medical Treatment of International Students in Nanchong. Open Access Library Journal, 8: e8067. https://doi.org/10.4236/oalib.1108067

Received: October 12, 2021

Accepted: November 23, 2021

Published: November 26, 2021

Copyright (๑) 2021 by author(s) and Open Access Library Inc.

This work is licensed under the Creative Commons Attribution International License (CC BY 4.0).

http://creativecommons.org/licenses/by/4.0/

\begin{abstract}
To investigate and provide better medical service for the international students in Nanchong, 131 international students who sought for medical service in the Second Affiliated Hospital of North Sichuan Medical College from April 2018 to March 2021 were selected as the research subjects, one questionnaire with 25 questions on routine physical examination, medical satisfaction, medical expense, service quality and medical assistance was conducted, then, the results were analyzed by SPSS 22.0. According to the results, the international students encountered some difficulties in the process of seeking medical service, to solve these difficulties and provide better medical service for the international students, the collaborations of the enrollment units, the medical institutions and the international students are needed.
\end{abstract}

\section{Subject Areas \\ Public Policy, Sociology \\ Keywords \\ International Students, Medical Condition, Medical Investigation \& Reflection}

\section{1. 引言}

自 1950 年第一批来华留学生至今, 我国的留学生培养工作已有 70 年的 历史。留学生的学习内容也经历了从开始的单纯的汉语学习发展到当下涵盖 各学各科各领域的全盛局面[1]。来华留学生的培养工作不仅是国家文化软实 力的一大体现, 也是我国的国际地位不断攀升、国际影响力持续增长的最好 名片 [2]。南充市作为四川省第二大高等教育集中地, 其留学生招生工作已有 
15 年的历史, 共计培养医学本科生、研究生 555 人。留学生招生单位和南充 市医疗机构担负着为留学生的身心健康保驾护航, 帮助他们顺利完成学业的 重责。本文对南充市川北医学院第二附属医院近 3 年以来收治的外籍患者进 行问卷调查分析, 在对该地区的留学生就医状况进行分析的同时, 为其医疗 单位涉外医疗服务水平的改进和提升提供参考依据。

\section{2. 调查对象与方法}

\section{1. 调查对象}

以南充市川北医学院第二附属医院 2018 年 4 月至 2021 年 3 月收治的 131 名留学生为调查对象展开研究, 有效研究对象 126 人。

\section{2. 方法}

自制《留学生就医状况调查研究问卷》对近 3 年以来在川北医学院第二 附属医院接受过医疗服务的留学生进行问卷调查。为保障研究的客观性和科 学性, 问卷以中英文、选择题的形式编写, 问卷内容由 5 大板块(常规体检, 就医体验, 医疗费用, 服务质量, 医疗援助)、23 个问题组成, 从语言、医疗 服务、设施设备、医疗费用等不同角度收集留学生的就医信息和就医体验。 经测试, 问卷的信度和效度分别为 0.732 和 0.727 , 所得结果能作为数据分析 依据。随后, 对收集到的调查结果运用 SPSS 22.0 软件进行分析、整理。本次 调查共计发出问卷 131 份, 回收有效问卷 126 份, 有效率为 $96.18 \%$ 。

\section{3. 结果}

在回收的 126 份有效问卷中, 男性 54 例, 女性 72 例, 年龄主要集中分布 在 19 22 岁之间(占比达 46.03\%); 亚洲的留学生占比高达 $73.02 \%$ (其中印度 和巴基斯坦的就诊比例分别为 $21.43 \%$ 和 $26.98 \%$, 占总就诊人数的三分之二), 非洲和欧洲的留学生的就诊占比分别为 $23.01 \%$ 和 $3.97 \%$ 。主要调查结果如下:

\section{1. 常规体检方面}

参加本次调研的所有留学生对招生单位为所有留学生在入校前组织常规 体检的举动表示赞赏, 但 $95.24 \%$ 以上的留学生认为, 增加体检频率, 如两年 一次、一年一次的体检活动更能保障他们的身心健康。同时, $91.27 \%$ 以上的 研究对象认为, 常规体检的项目选项方面可提供个体自选项目, 以便他们能 更好的了解自己的身体健康状况或是进行有针对性的健康预防保障工作。结 果见表 1 。

\section{2. 就医体验方面}

1) 语言方面。虽然留学生从一年级开始学习汉语, 但 $89.67 \%$ 以上的留学 生认为他们在课堂上学习到的汉语并不足以帮助他们在就医过程中使用汉语 和医务人员进行有效的交流和沟通。因此, 英语成为留学生就医的重要语言 工具, 但因使用者存在口音和语气等差异, 因语言障碍带来的交流不畅成为 留学生就医体验不佳的一大因素[3]。 
2) 医疗环境和医疗设施方面。 $88.89 \%$ 以上的留学生认为就诊医疗单位 的医疗设施及设备能为他们的医疗服务提供足够的设备保障, 但 $69.84 \%$ 留 学生认为, 医疗单位应为外籍学生就诊提供更加私密的医疗环境, 避免就诊 室其他患者的 “注目礼” 或因生活和文化差异造成的同病房塩䛈[4]。结果 见表 2 。

表 1. 南充市留学生体检调查情况

\begin{tabular}{|c|c|c|c|}
\hline 板块 & 主题 & 选项 & 百分比(\%) \\
\hline \multirow{2}{*}{ 入校体检 } & \multirow{2}{*}{ 合理性 } & 合理 & 100 \\
\hline & & 不合理 & 0 \\
\hline \multirow{15}{*}{ 常规体检 } & \multirow{4}{*}{ 频度 } & 4 年/次 & 13.49 \\
\hline & & 2 年/次 & 16.67 \\
\hline & & 1 年/次 & 9.52 \\
\hline & & 0 年/次 & 60.32 \\
\hline & \multirow{2}{*}{ 合理性 } & 合理 & 19.05 \\
\hline & & 不合理 & 80.15 \\
\hline & \multirow{4}{*}{ 期望值 } & 4 年/次 & 4.76 \\
\hline & & 2 年/次 & 36.51 \\
\hline & & 1 年/次 & 58.73 \\
\hline & & 0 年/次 & 0 \\
\hline & \multirow{2}{*}{ 体检项目 } & 体检套餐 & 8.73 \\
\hline & & 自选项目 & 91.27 \\
\hline & \multirow{3}{*}{ 费用渠道 } & 招生单位 & 66.67 \\
\hline & & 自己承担 & 9.52 \\
\hline & & 招生单位和自己分担 & 23.81 \\
\hline
\end{tabular}

表 2. 南充市留学生就医体验调查情况

\begin{tabular}{|c|c|c|c|}
\hline 板块 & 主题 & 选项 & 百分比(\%) \\
\hline \multirow{5}{*}{ 语言 } & \multirow{2}{*}{ 是否困难 } & 困难 & 89.67 \\
\hline & & 不困难 & 10.32 \\
\hline & \multirow{3}{*}{ 困难原因 } & 口音或语气 & 65.87 \\
\hline & & 文化背景差异 & 21.43 \\
\hline & & 表达方式差异 & 12.70 \\
\hline \multirow{4}{*}{ 医疗环境 } & \multirow{2}{*}{ 是否满意 } & 满意 & 30.16 \\
\hline & & 不满意 & 69.84 \\
\hline & \multirow{2}{*}{ 不满意原因 } & 环境不适应 & 37.30 \\
\hline & & 缺乏个人隐私 & 62.70 \\
\hline \multirow{4}{*}{ 医疗设施 } & \multirow{2}{*}{ 是否满意 } & 满意 & 88.89 \\
\hline & & 不满意 & 11.11 \\
\hline & \multirow{2}{*}{ 不满意原因 } & 医疗器械不足 & 30.95 \\
\hline & & 医疗设备老化 & 69.05 \\
\hline
\end{tabular}




\section{3. 医疗费用方面}

我市的留学生均由招生单位牵头, 购买商业保险, 因就诊原因、就诊途 径不同, 报销比例也有所不同; 如, 门诊就医留学生承担费用比例较高, 住 院就医按照国家相关规定可通过商业保险报销一定比例的医疗费用。目前大 部分生源国未提供医疗费用报销, 招生单位出于人文关怀原则给与住院学生 一定的慰问金。就医疗总开支而言, 检查费占比最高, 其次为药费和床位费。 结果见表 3。

\section{4. 服务质量方面}

排除语言因素， $85.71 \%$ 以上的留学生对医疗单位的医务人员所提供的医 疗服务持肯定态度, 认为 “医务人员笑容和善, 耐心真诚。” 但仍有 $9 \%$ 的少 数医务人员因就诊患者较多时 “耐心不足” 或 “存在赶时间的嫌疑。” 结果 见表 4。

\section{5. 医疗援助方面}

虽然医疗单位所有的窗口和楼层均提供中英文的语言向导，但未有外籍 人士就医的就诊指南或说明; 目前尚未设置涉外医疗咨询窗口或开设外籍诊 疗部门, 但 $18.25 \%$ 的留学生表示接受过语言援助, 此板块的统计差异与就诊 科室相关。结果见表 5 。

表 3. 南充市留学生医疗费用调查情况

\begin{tabular}{|c|c|c|c|}
\hline 板块 & 主题 & 选项 & 百分比(\%) \\
\hline \multirow{12}{*}{ 医疗费用 } & \multirow{4}{*}{ 承担渠道 } & 生源国 & 5.56 \\
\hline & & 招生单位援助 & 16.67 \\
\hline & & 商业保险 & 28.57 \\
\hline & & 本人承担 & 48.41 \\
\hline & \multirow{3}{*}{ 开支情况 } & 检查费 & 53.17 \\
\hline & & 药品费 & 33.33 \\
\hline & & 床位费 & 13.49 \\
\hline & \multirow{2}{*}{ 是否意度 } & 满意 & 26.98 \\
\hline & & 不满意 & 73.04 \\
\hline & \multirow{3}{*}{ 不满意原因 } & 医疗费用高 & 60.32 \\
\hline & & 报销比例低 & 21.43 \\
\hline & & 报账周期长 & 18.25 \\
\hline
\end{tabular}

表 4. 南充市留学生医疗服务质量调查情况

\begin{tabular}{cccc}
\hline 板块 & 主题 & 选项 & 百分比 $(\%)$ \\
\hline \multirow{3}{*}{ 医疗服务质量 } & 满意 & 耐心、真诚 & 62.04 \\
\cline { 2 - 4 } & 不满意原因 & 笑容、和善 & 37.96 \\
& & 耐心不足 & 61.11 \\
& & 赶时间 & 38.89 \\
\hline
\end{tabular}


表 5. 南充市医疗单位涉外医疗援助调查情况

\begin{tabular}{|c|c|c|c|}
\hline 板块 & 主题 & 选项 & 百分比(\%) \\
\hline \multirow{10}{*}{ 医疗援助 } & \multirow{2}{*}{ 语言向导 } & 窗口向导 & 100 \\
\hline & & 楼层向导 & 100 \\
\hline & \multirow{2}{*}{ 就医指南或说明 } & 满意 & 0 \\
\hline & & 不满意 & 100 \\
\hline & \multirow{2}{*}{ 涉外咨询窗口 } & 开设 & 0 \\
\hline & & 未开设 & 100 \\
\hline & \multirow{2}{*}{ 涉外诊疗部门 } & 开始 & 0 \\
\hline & & 未开设 & 100 \\
\hline & \multirow{2}{*}{ 语言援助 } & 开设 & 18.25 \\
\hline & & 未开设 & 81.75 \\
\hline
\end{tabular}

\section{4. 问题分析}

\section{1. 留学生医疗意识不足}

我市的留学生来自世界 20 多个国家和地区, 存在语言多样性、文化多元 化、宗教信仰多源化等情况。虽然招生单位配备了经验丰富的专职教师和生 活老师帮助留学生适应新环境中的教育、教学和管理工作, 但留学生在就医 方面依然存在着诸多问题。如, 留学生不熟悉当地的就医流程, 耽误就诊时 间; 留学生对招生单位依赖性过强, 缺乏积极主动的医疗意识。

\section{2. 医务人员外语能力尚待提高}

语言是交流沟通的一把钥匙 [5]。在医疗服务中, 医务人员不仅肩负着帮 助病人消除身体病痛的责任, 还兼负着宽慰病人紧张心理的重责。异国就医, 医务人员若是语言交流能力不足, 难以宽慰病人的紧张心理、卸下患者的心 理防备, 不利于医疗服务的顺利开展。

\section{3. 医疗单位涉外接诊能力亟需提升}

虽然近年以来我国政府为了保障广大人民群众的身体健康投入了大量资 金用于先进医疗设备的购买和医疗设施的配备, 但是我市医疗单位的涉外医 疗能力依然相对滞后。如, 我市尚未有医疗单位提供专人、专岗的涉外医疗 服务; 医疗单位对医务人员的外语能力、涉外文化传统、风俗习惯、宗教信 仰等重视度不够、培养力不足。

\section{4. 医疗费用解决渠道不太理想}

$73 \%$ 以上的留学生反映, 异国就医存在 “医疗费用高”、“报销比例低”、 “报账周期长”、“手续繁琐” 等问题。其自身的经济条件成为留学生寻求 医疗服务的一大障碍 [6]。尤其是在实际的医疗服务中, 门诊就医的几率大于 住院就医, 商业保险对于门诊医疗的费用解决意义有限。再者, 我市留学生 商业保险的报账时间为每年的 6 月和 12 月, 对于大部分来自欠发达国家或地 
区的留学生生活带来不小的经济负担。

\section{5. 留学生的健康意识不强、自我保护能力不足}

我市留学生 70\%以上来自于亚热带或热带地区, 在饮食习惯、服饰穿戴 上与我市差异较大。所以即便是在寒冷的冬天, 依然可见留学生穿着单薄的 衣物, 更有少数留学生保险意识不强, 拒绝自愿购买保险, 缺乏自我保护意 识。

\section{5. 建议}

\section{1. 留学生应树立积极主动的健康意识}

入校前, 留学生应积极主动地查询招生单位所在地的气候类型和季节特 点, 避免校园内夏季穿着冬季衣物, 冬季穿着夏季衣物的尴尤局面; 入校后, 留学生应树立长远的健康意识, 积极主动地熟悉就近的医疗机构、学习就医 流程; 开展留学生互助小组活动, 在适应学校生活的同时, 组织健康服务小 团队, 利用所学知识, 为自己的健康保驾护航。

\section{2. 医务人员的自我提升}

鼓励医疗机构各科室的医务人员通过在线课堂、线下听课、高校教师互 帮互助等多种形式纠正已有不规范语音、提高自己的外语口语水平, 能熟练 运用外语为留学生提供常见疾病的诊疗工作, 弥补外籍患者因语言交流障碍 而造成的 “医疗信任度不足”。

\section{3. 医疗单位涉外就诊部门的开设}

医疗国际化时代已拉开序幕，为了和国家的医疗国际化相接轨，我市目前 已规划国际医院。遗憾的是, 我市留学生的招生工作已有 15 年之久, 我市的 三级综合医院却尚未开设外籍诊疗部门或外籍诊疗窗口。诚然，外籍诊疗部门 或外籍诊疗窗口的开设对从业人员的语言能力、业务能力等均有较高要求，外 籍诊疗部门的开设是一个地区综合医疗能力的有力证明, 也能为一个地区医疗 国际化的发展提供第一手的经营和管理经验，助力地区的医疗国际化。

\section{4. 留学生保险意识的加强或提升}

国家主席习近平指出, 我国政府始终关注留学生的身体健康, 并对留学 生给予一视同仁的关心和爱护。各招生单位对来华留学生提供了多种形式的 奖学金或助学金, 留学生应树立正确的金钱意识、做到防患于未然, 积极主 动地购买商业保险, 为自己的健康提供多充保障。

\section{5. 留学生责任意识的培养}

留学生是自我健康的第一责任人, 应认识到自身行为对自我健康的重要 影响。一方面, 留学生应树立健康意识, 积极参加体育锻炼, 提高自身体能 及对疾病的免疫能力; 另一方面, 留学生应积极地适应新的学习和生活环境、 
认真地学习汉语, 提高自己的语言运用能力, 能用普通话或是当地方言对常 见疾病进行描述, 做自我健康的第一保护人。

\section{6. 招生单位对留学生身心健康的持续关注}

作为留学生在华期间扮演 “家” 这个角色的招生单位来讲, 应引导留学 生认识到其身体健康的重要性、为留学生在入校前和入校后提供持续的健康 关爱活动; 通过在校学生和留学生在文化、学习、体育等方面的交流和文娱 活动为留学生提供更多的语言学习机会, 夯实就医语言; 鼓励留学生学习中 医在养生和保健方面的知识, 在发扬中国传统医学的同时, 防患疾病于未然。

\section{6. 结语}

我市留学生对医疗机构提供的医疗服务总体满意程度较高, 但在实际的 就医过程中, 仍存在一定的语言交流障碍; 其次, 留学生对招生单位和医疗 单位的期望值较高, 但其自身的健康意识、自我保护意识和抵御疾病风险意 识又较为薄弱, 自我健康防护能力有待加强; 再次, 医疗单位的涉外接诊能 力仍有提高和改进空间, 招生单位对留学生健康意识的培养和关注也需继续 保持。诚然, 地区之间存在医疗差异, 本文仅能代表本市留学生在就医时遇 到的问题, 因此具有一定的局限性, 但总体来讲, 目前研究者对留学生在就 医或医疗服务方面的研究较为匮乏, 为留学生提供优质的医疗服务还需更多 的研究数据和依据, 也需要更多的研究者参与其中, 共享经验和成果。

\section{基金项目}

南充市社科研究 “十三五” 规划 2020 年度青年项目 (项目编号: NC2020C070); 南充市社科研究“十三五”规划 2017 年度青年项目(项目编号: NC2017C045)。

\section{Conflicts of Interest}

The author declares no conflicts of interest.

\section{References}

[1] 杨春耘, 严宏伟. 临床医学留学生对外汉语教学的反思[J]. 云南师范大学学报 (对外汉语教学与研究版), 2018, 16(4): 38-43.

[2] 费维, 房良. 基于全球卓越城市建设背景下的上海市涉外医疗发展研究 [J]. 卫生 软科学, 2019, 33(7): 33-38.

[3] 刘春霞, 邹绍选. 红河学院留学生在校满意度调查 [J]. 文教资料, 2021(1): 138-139+151.

[4] 凌丹玥, 严玉茹. 留学生就医满意度的调查与分析[J]. 中西医结合护理(中英文), 2019, 5(6): 53-56.

[5] 杨扬. 来华留学生跨文化适应研究——以杭州地区部分高校为[J]. 文化创新比较 研究, 2018(3): 193-194.

[6] 李艳迪. 开设外籍门诊在外籍患者管理中的应用效果 [J]. 中医药管理杂志, 2015, 23(17): 92-94. 


\section{Appendix (Abstract and Keywords in Chinese)}

\section{南充市留学生就医情况调查与思考}

摘要: 为了解南充市留学生的就医情况, 为南充市留学生提供优质的医疗服 务, 本文以 2018 年 4 月至 2021 年 3 月在川北医学院第二附属医院就诊的 131 名留学生为研究对象, 对其就接受过的医疗服务的五大板块、 25 个选项进行 问卷调查, 并用 SPSS 22.0 软件对调查结果进行数据分析。结果显示, 南充市 留学生在就医过程中依然存在一定的就医障碍, 解决留学生的就医问题、为 留学生的学习提供健康保障需要招生单位、医疗机构和留学生的三方合力。

关键词: 留学生, 就医状况, 就医调查及思考 